\title{
An Option of Hybrid Virtual Labs in an Introductory Biology Course as the Means for Accessible Learning and Enhancing Student Education
}

\author{
Kyle Bactol, Andrew E. Laursen, Charlotte de Araujo \\ Department of Chemistry and Biology, Ryerson University, Toronto, Canada
}

\begin{abstract}
A virtual lab can be defined as a laboratory exercise performed outside of the physical laboratory. One interpretation of this includes partially virtual or take-home labs where students are led through online video demonstrations while physically conducting the experiment with a lab kit. Here we describe a hybrid virtual lab designed and implemented for undergraduate biology which offers a combination of take-home laboratory assignments coupled with in-laboratory sessions. It is an alternate option for introductory biology students which provides an opportunity to accommodate different learning styles, preferences, or needs. Although not an exhaustive list, these needs might arise from competing commitments to family, employment, sports, or the toll of long commutes. Offering flexibility in laboratory format may allow a student to better manage these conflicts and improve the quality of the overall university experience. Students retaking the biology laboratory were given an option of participating in conventional laboratories or the hybrid format. Benefits or costs of choosing between these lab formats are determined by examining the scores of students on assessments that test laboratory skills and knowledge. Performance was compared between the two groups of students through an analysis of covariance (ANCOVA), using previous laboratory scores as a covariate. Ultimately, researching virtual lab effectiveness as an alternate teaching method may create a dialogue between curriculum developers and institutions to discuss how to implement them as a way to enhance the overall student learning experience.
\end{abstract}

\section{Introduction}

These guidelines include complete descriptions of the. Physical laboratories present the opportunity for science students to develop many practical scientific skills such as: interacting directly with specialized equipment, experiencing data collection, handling samples, and applying theoretical knowledge to real world situations [1]. The advances in computer technology over the past two decades has allowed educators to simulate a physical laboratory with various materials and equipment to the point where students can virtually conduct scientific experiments
[2]. However, virtual laboratories have lagged in biology, as the physical setting of a biology laboratory, and the physical manipulations that are part of the skill set to be developed, cannot easily be simulated.

The vague interpretations and definitions of a virtual lab create many different online learning simulations within the literature collectively identified as "virtual labs". However, there are three types of virtual labs that are most commonly discussed in pedagogical research: remote labs, completely virtual/simulated labs, and supplemented virtual labs. In remote labs, students could conduct experiments outside of the physical lab over the internet by utilizing web applications which enable real equipment in a lab to adjust directly to feedback from the inputs and commands of the user [3]. An example of this interaction would be a student controlling a $\gamma$-ray spectrometer to analyze different elements and isotopes [3]. A potential benefit of remote labs is the reduced cost of having or maintaining specialized equipment to the institution. Students can book time slots to conduct experiments and effectively share the remote lab [4]. This approach may provide students with a good fundamental understanding of the experiments conducted, however it does not provide students with practical lab experience, nor does it develop the manipulative skills collectively referred to as "good laboratory hands". As Beverly Park Woolf, computer scientist at the University of Massachusetts Amherst stresses, students should handle the equipment, measure out a reagent, or tweak a dial [3].

In a completely virtual/simulated lab, the student conducts an experiment that is based entirely on animations, web applications, and user inputs to adjust different parameters in order to observe theoretical results with visual representations on a particular piece of technology (e.g. computer, smartphone, tablet) without coming into contact with real equipment or samples [5]. One such instance is the use of a virtual physiology of exercise laboratory program at the University of Florida (Department of Applied Physiology ad Kinesiology). Students at this institute had simulated test designs and data collection of various physiological modules as opposed to traditional hands-on lab activities [6]. The merit of this approach to virtual labs is that well 
designed software can be easily distributed to a large number of students while completely bypassing the need for purchasing or maintaining specialized equipment [7]. Another positive aspect of this type of laboratory is that there are no boundaries to the experiment that could be conducted. In the virtual word, reagent and equipment costs impose no limits, and vast spatial and temporal time scales are not barriers. However, a distinct shortcoming to this approach is the lack of practice in handling equipment and development of laboratory skills. A remote lab has some level of simulating real laboratory experience where students can feel like they are physically conducting the experiment; a completely virtual lab does not have this representation of the physical laboratory environment.

The third type of virtual labs are supplemented virtual labs. These blend some of the simulation aspects of virtual labs, with physical experiments using take-home laboratory kits. Kits contain various equipment and materials that students must use to complete the experiments [8]. For example, students enrolled in science courses at Open University in Milton Keynes, UK have kits that might include microscopes, chemistry sets, or lasers that would be used to conduct experiments at home [3]. A merit of this approach is that the lab kits provided to students compensate the lack of handling specialized lab equipment [8]. Conversely, with a large group of students or lack of sufficient funding, providing lab kits to science students has been found to be an expensive and inefficient process [3]. This, of course, will depend on the content of the kits and the nature of the experiments.

\section{Incorporating Virtual Labs into Science Curriculum}

There are various factors that facilitated the introduction of virtual labs into science education. Introductory science courses tend to have very large enrollment and it is often difficult or resourceintensive to provide opportunities of physical scientific investigation for all the students [9]. Virtual labs present a mode of delivery for institutions that allows reduced spending on equipment and teaching staff, with fewer physical resources required. The self-guided nature of virtual labs, coupled with extensive use of video demonstrations, can reduce the number of staff required relative to conventional laboratory instruction. Another advantage of virtual labs is the added flexibility in scheduling. Laboratories are typically scheduled in three hour blocks which may create scheduling conflicts for other courses a student might wish to take, and which constrains the availability of teaching laboratories and potential alternate uses of space. Conventional labs also have a pre-determined schedule and in the case of students missing these labs for any reason, it is often difficult for the professor to arrange for a makeup lab [7]. Virtual labs allow students to conduct experiments that would not otherwise be possible due to the hazardous nature of materials or intensive training required to safely use equipment. This may be particularly true in chemistry education. In addition to lowering safety and training barriers, virtual labs unlock the opportunity for students to investigate various scientific phenomenon that are not feasible in a physical lab, benefiting from the advancements in computer technology and simulations [10]), for example the use of software which allows students to inquire and observe diffusion and osmosis at the molecular level [11].

To be clear, the authors are not advocating replacement of all conventional laboratories with virtual labs. There will be certain skills that can only be acquired through practice with the relevant equipment, and under close supervision by welltrained instructors. However, there will be courses in which a virtual lab may be a viable format. Different models of virtual labs are best suited to different circumstances. A completely virtual lab may work best within an ecology or bioinformatics course where many concepts are difficult to model or observe in real-time. A remote lab may be the best option for an optics course where students can almost feel like they are handling lasers, mirrors and other equipment used to investigate different physical phenomenon. A supplemented or hybrid virtual lab may be best suited to a basic biology course where development of basic, practical skills is very important, but students are typically not yet using advanced techniques or high-end scientific equipment. These basic skills might be attained using relatively pedestrian equipment that could be supplied in a kit.

\subsection{Results of Implementing Virtual Labs}

The majority of pedagogical studies indicates that the use of virtual labs in science courses have positive results. One aspect of assessing virtual labs are student preferences, and several studies have documented student preference for virtual labs over traditional labs [8], [9], [12], [13]. A student may prefer the virtual lab for a variety of reasons, but whatever the reason, offering a virtual lab option would appear consistent with a learning environment that responds to the desires and needs of students. However, it must be noted that there are studies which reported students perceiving virtual labs negatively over a conventional lab setting [14]. Student preference notwithstanding, a concern in offering a virtual lab format is whether it develops comparable laboratory and/or research skills to a conventional lab. Will students completing virtual 
labs perform as well as students completing conventional labs? Will course content be reinforced as effectively, and will the student develop the same necessary skills to succeed in a science program? This facet of success for virtual labs has been less well characterized than has student preference. One study reported a positive impact on student performance in a physical chemistry course [15]. A computer-generated viscosity simulation was used as a pre-laboratory supplement, and the group of students who conducted the virtual lab in-lab experiments outperformed the students who only conducted the in-lab experiments on a knowledgebased questionnaire (simulation students with mean scores 23 to $29 \%$ higher than the non-simulation students) distributed at the end of the course in order to evaluate their understanding of theory applied during the experiments [15]. Comparing success of students completing different formats of a general biology laboratory is a focus of the current study, as institutions must be confident students learn effectively before substituting virtual labs for conventional labs.

\subsection{Research Objectives and Rationale}

This research considers the outcome of a pilot project offering students the same laboratory experience via different delivery modes. At the local program level, this could affect the approach taken within the program for delivering laboratories in some, although certainly not all, biology courses. If offering alternative delivery modes is successful, from dual perspectives of student achievement and student desire, this may have implications for the quality of the undergraduate experience, program resource allocation, and scheduling/use of physical space. The research will foster discussion on whether hybrid laboratories ought to be considered for a broader rollout. Beyond the Ryerson University program, the effectiveness of a hybrid virtual lab as an alternative teaching method may create a dialogue at other institutions to discuss the means of implementing such labs to enhance the overall student learning experience.

One of the reasons these hybrid virtual labs were initially developed was to accommodate different student learning styles and needs. There is no one perfect way to develop laboratory and research skills as the individual strengths and weaknesses may vary immensely between a student and her peers [16]. Beyond developing these skills, a laboratory also reinforces content learned in a lecture, and the approaches that most effectively reinforce lecture content will also vary among students. Offering students options and allowing them to select laboratory formats that best meet their needs may lead to better outcomes than requiring all students learn in the same mode.
The typical undergraduate student may have commitments outside of their academic program which may include but are not limited to: family, employment, sports, clubs, and relationships. In addition to this, there may be external factors such as: commuting distance, level of comfort with technology, and approach to learning that affect their university experience. The option to choose a hybrid virtual lab or a conventional lab for an undergraduate biology course would allow for greater flexibility in addressing the different needs of students enrolled. Technology serves an integral part of student life both inside and outside the classroom where almost every student uses a smartphone, laptop or tablet on a regular basis. Advances in technology can allow changes in how we deliver content or approach learning, and we can adapt to the needs of the current student population and future generations with the overall goal of improving student learning outcomes. Offering virtual labs in an undergraduate science course is an example of such adaptation that may improve learning outcome if used deliberately and with clear vision of the learning objectives for the course. Ultimately, this pedagogical research is a step towards determining if a non-traditional lab format can accommodate students without compromising the quality of the content and skills development relative to a traditional lab format.

\section{Methods}

This study assessed if the Ryerson University hybrid virtual lab approach has merit as an alternate form of delivery. The overall goal of this project was to determine the effectiveness and value of Ryerson biology virtual labs as assessed through student performance. The assessment of hybrid virtual labs used quantitative data and student lab performance indicators, to consider the value of this format relative to conventional laboratories.

\subsection{The Ryerson Context for Hybrid Virtual Labs}

For this study, the hybrid virtual labs were developed at Ryerson University for implementation in the introductory biology course: Biology I - BLG 143. This is a mandatory introductory course providing incoming university students with foundations in biology. The course consists of two components: lectures totaling three contact hours per week for twelve weeks, accompanied by bi-weekly three hour laboratories. BLG 143 is offered in two semesters. The first offering during the fall semester, typically has enrolments of 600-700 students. Students who were unsuccessful in their first attempt in Biology I, or who dropped the course for any reason have an opportunity to attempt the course again in the second offering (winter semester), 
referred to as a transition offering. Course enrolment during the Winter, 2015 transition offering was 25 undergraduate students. While the content and testing strategies are identical, the second offering of BLG 143 offers a smaller student-instructor ratio in the lecture.

Laboratories end after the tenth week of the semester. Given the late drop deadline (after the tenth week), most students taking Biology I in transition have previously completed the Biology I laboratories, even if not completing the course for credit. This creates a pool of students in the transition offering of biology who have a previous record of performance in the laboratory against which laboratory performance during the transition offering can be compared. Of the 25 students enrolled in Winter, 2015 transition, 23 had completed the laboratory in Fall, 2014. All 25 of these students were provided with information regarding the hybrid virtual lab offering and given the option to select either the conventional laboratory or the hybrid format.

Of the three main types of virtual labs, Ryerson's hybrid labs most resembled supplemented virtual labs due to the inclusion of take-home lab kits which were to be used in conjunction with the video demonstrations and lab manual. The Ryerson hybrid labs differed from virtual labs described in the literature which were developed specifically as virtual labs with no parallel conventional labs. The Ryerson hybrid labs began with the conventional format laboratory manual, and protocols for activities and materials used were adapted to allow their safe completion using take home kits. Therefore, nearly all activities in the conventional lab offering had close parallel activities in the hybrid lab offering, and vice versa. The expectation was that students could develop the same laboratory and research skills through either offering since the activities were nearly identical. The principle difference was the physical setting in which the activities were completed.

Some studies have described virtual labs offered as optional resources to students in a science course [5], [9]. At Ryerson, students in the transition Biology I course were given the option to complete the laboratory either by the conventional format, or by the hybrid format. Students electing the conventional format did not also have access to the hybrid format resources as supplementary material. Students opting into either format ideally received the same quality of experience, and completed laboratories of similar difficulty, although the limited instruction of the hybrid format placed greater onus on the students. To compensate, the hybrid labs included comprehensive videos for demonstrating and explaining experiments akin to the training a TA would provide.

\subsection{Structure of Hybrid Virtual Laboratories}

The Biology I laboratory was offered in two formats, the traditional in-lab format and an alternative hybrid virtual lab. Both formats consisted of five modules each with specific objectives and key scientific concepts (see Table 1).

Table 1. Description of Hybrid Virtual Labs from Biology

1. Each lab had a specific focus or theme and often

incorporated multiple exercises or activities to demonstrate the key concepts in the initial readings of the module

\begin{tabular}{|c|c|}
\hline $\begin{array}{l}\text { Laboratory } \\
\text { Title }\end{array}$ & Objectives \\
\hline $\begin{array}{l}\text { Laboratory 1: } \\
\text { The Scientific } \\
\text { Method }\end{array}$ & $\begin{array}{l}\text { Understand and use the Scientific } \\
\text { Method. Correctly develop a null and } \\
\text { altemate hypothesis. }\end{array}$ \\
\hline $\begin{array}{l}\text { Laboratory 2: } \\
\text { Structure and } \\
\text { Function of } \\
\text { Proteins }\end{array}$ & $\begin{array}{l}\text { Understand primary, secondary, and } \\
\text { tertiary structure of peptides. } \\
\text { Understandinteractions between amino } \\
\text { acid side chains and carboxyl groups } \\
\text { that explain the secondary and tertiary } \\
\text { peptide structure. Understand why } \\
\text { chemical and physical environment } \\
\text { affect molecular structure. Understand } \\
\text { how chemical and physical } \\
\text { environments can affect pigment and } \\
\text { enzyme activity. }\end{array}$ \\
\hline $\begin{array}{l}\text { Laboratory } 3 \text { : } \\
\text { Microscopy }\end{array}$ & $\begin{array}{l}\text { Properly use a microscope and identify } \\
\text { all its parts. Describe and recognize the } \\
\text { stages of mitosis in plant and animal } \\
\text { cells. Distinguish between diffusion } \\
\text { and osmosis. Recognize the effects of } \\
\text { solutes on osmosis }\end{array}$ \\
\hline $\begin{array}{l}\text { Laboratory 4: } \\
\text { Metabolism }\end{array}$ & $\begin{array}{l}\text { Observe the effects of light intensity on } \\
\text { net photosynthesis. Understand the } \\
\text { difference between light dependent and } \\
\text { independent reactions. Recognize how } \\
\text { the TTC can be used to detect electron } \\
\text { transport in living organisms. }\end{array}$ \\
\hline $\begin{array}{l}\text { Laboratory } 5 \text { : } \\
\text { Introduction } \\
\text { to Genetics }\end{array}$ & $\begin{array}{l}\text { Understand the differences between } \\
\text { homozygous/ heterozygous traits, } \\
\text { phenotype and genotype. Predict } \\
\text { potential genotypes based on } \\
\text { phenotypes. Apply understanding of } \\
\text { genetics to predict outcomes of specific } \\
\text { crosses. Recognize differences in } \\
\text { inheritance patterns between linked } \\
\text { traits and independently assorting } \\
\text { traits. Determine the allele frequency } \\
\text { after specific events. }\end{array}$ \\
\hline
\end{tabular}

These labs reinforced concepts covered in lecture. For instance, Lab 1 introduces students to fundamental laboratory skills (such as hypothesis testing), this concept is repeated throughout the series of labs to strengthen their retention. Students 
in the hybrid lab were provided with a take-home kit with materials/reagents to carry out their experiments independently (see Table 2). Equivalent resources were provided to students who participated in the conventional lab, with some modifications. For example, in Lab 3 students completing the hybrid virtual laboratory format used the SmartMicroscope application (www.smartmicrophone.net)_an online application to learn basic principles of microscopy and to study various aspects of the cell cycle. These students also watched a youtube video on use of light microscopes. They were then required to schedule a time to come into lab and demonstrate skills using a microscope, working with the instructor. In Lab 4, methanol and hot plates were provided to students in the conventional lab setting for extraction of pigments from plant leaves. In the take-home kits, no methanol was provided. Instead the protocol was modified to use a different plant with pigments easily extractable in hot water, and students stopped by the laboratory the week of the lab to obtain plant leaves to work with at home. An experiment in the conventional laboratory used triphenyl tetrazolium chloride (TTC) to observe electron transport activity in plant embryos. In the hybrid virtual format, TTC was not supplied to students and this activity was replaced by a video demonstration.

Students completing labs in the conventional format had three-hour lab sessions. Students enrolled in the hybrid virtual lab were not restricted to three hours, but had deadlines for posting their laboratory results, including posting videos or photos of their experimental results.

Assessment for both laboratory formats included bi-weekly lab quizzes, bi-weekly lab report sheets on modules, a written formal report, and a final laboratory exam. These quizzes were completed within a limited time period (10 minutes). In the conventional lab setting, these were administered at the start of the lab session. In the virtual lab format, there were administered online. Multiple versions of quizzes were used for both online and in-class assessments. For a student completing quizzes online, the version available would be randomly assigned. Students would have a designated time to log onto the course management system and complete the quiz. The lab report sheets required students to record their observations and answer related questions about the experiments they performed. For instance, students in lab two answered questions about the effects of physical environment on protein folding (Table 1). Students in the conventional laboratory setting would fold their model and show their TA for verification. Students completing the hybrid virtual lab would produce a video showing the folded model with rotation and submit the video to their TA.
Table 2. Reagents and materials provided in take-home kit for hybrid virtual lab participants from Biology I. Each lab had specific materials for students to complete the exercises.

\begin{tabular}{|c|c|}
\hline $\begin{array}{c}\text { Laboratory } \\
\text { Title }\end{array}$ & Take-home kit supplies \\
\hline Laboratory 1 & - Eight "Jumping Beans" \\
\hline Laboratory 2 & 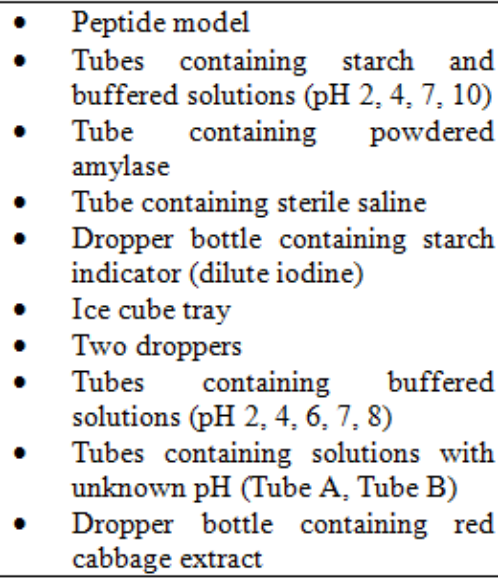 \\
\hline Labor & - Smart Microscope application \\
\hline Labo & 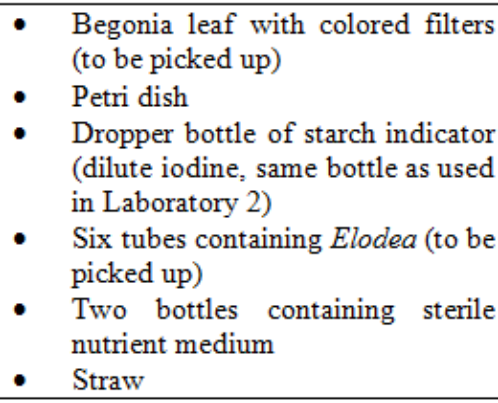 \\
\hline Laborator & $\begin{array}{l}\text { - } \text { Peptide model "backbone" } \\
\text { - Colored rings } \\
\text { - Envelope with Genetics Problems }\end{array}$ \\
\hline
\end{tabular}

The formal report introduced students to a scientific paper. They were required to answer questions regarding a scientific paper, particularly the structure of the paper. The objective was to help students understand how these papers are constructed through critical assessment, before they begin writing such papers. As this is an introductory course, the report also aimed to help students become more familiar with scientific jargon.

The final cumulative assessment was the lab exam, which was more focused predominantly on applying the information. The lab exam had both a written theory component and a practical component where students were required to demonstrate skills or demonstrate knowledge of concepts based upon things they had previously observed in the lab. All students, regardless of format, completed this as an in-lab exam. 


\subsection{Effectiveness of Virtual Labs}

The Biology I hybrid virtual lab pilot ran during the Winter, 2015 transition offering. Of the 25 students enrolled in the course, 2 were not included in analysis as they did not complete the laboratory in the previous fall. Of the remaining 23 students, 7 elected to complete labs using the hybrid virtual lab format, with the remaining 16 completing the conventional labs. The effect of laboratory format on student performance was assessed using analysis of covariance (ANCOVA). In the ANCOVA model, Fall 2014 laboratory scores were used as a covariate, to control for previous laboratory performance as a possible predictor of student achievement in Winter 2015. ANOCOVA models were run using overall lab scores (Winter 2015 as dependent variable and Fall 2014 as covariate), and also with quiz scores (again, Winter 2015 quiz average as dependent variable and Fall 2014 quiz average as covariate).

The overall lab score included some relatively subjective component marks, such as lab report sheet scores and laboratory paper scores. In Fall 2014, students might have had any of 14 different TAs, each with his own marking bias. However, the overall lab score was also heavily influenced by the lab exam, which included both fundamental knowledge and demonstration of laboratory skills. It was therefore useful as an indicator of whether students in the hybrid virtual lab group developed skill sets comparable to those of their classmates in the conventional lab group. Lab quiz averages were based on knowledge and comprehension based questions (Bloom's Taxonomy of Cognitive Learning Outcomes). The analysis of lab quiz averages helped distinguish students based on more strictly objective student assessments. SAS (Statistical Analysis Software) Enterprise Guide 6.1 was used to perform ANCOVA. Data were transformed as necessary to meet the assumptions of parametric statistical tests, specifically overall lab performance scores were power transformed (squared) to provide a data set with equal variance between treatment groups.

\section{Results and Discussion}

The laboratory format did have a significant effect on the overall laboratory scores for students in Winter $2016\left(\mathrm{~F}_{1,20}=7.62, \mathrm{p}=0.012\right)$. Students completing the hybrid virtual lab students scored $15 \%$ higher in the laboratory than students completing the conventional laboratories $(84 \% \pm 4 \%$, versus $69 \% \pm 3 \%$; mean \pm standard error) (figure 1 ). A student's previous performance in the lab during Fall 2014 was not a significant predictor of success in Winter $2015\left(\mathrm{~F}_{1,20}<0.01, \mathrm{p}=0.986\right)$. Students contemplating dropping the course may have stopped putting forward effort at different times during the semester. Performance in Fall 2014 may have reflected this approach from students rather than student ability to perform well in the course.

Student performance on laboratory knowledge and comprehension was similarly higher for the group completing hybrid virtual labs $\left(\mathrm{F}_{1,20}=3.68, \mathrm{p}=\right.$ 0.044 ). Students in the virtual lab section scored $\sim 14 \%$ higher than students in the conventional lab section $(87 \% \pm 3 \%$, versus $74 \% \pm 3 \%$; mean \pm standard error). As with overall lab score, a student's quiz average in Fall 2014 was not a significant predictor of success in Winter $2015\left(\mathrm{~F}_{1,20}=2.72, \mathrm{p}=\right.$ 0.074).

There are many possible explanations for the stronger performance of students opting for the hybrid virtual lab format. Perhaps this format facilitated better learning of the material. Perhaps it provided students with more time, or more flexibility in when they completed labs, and this helped performance. Or alternatively it relates to a difference in characteristics of students who opted in or out of the virtual lab. A larger sample size will be necessary to make more meaningful conclusions, as only a small number of students were enrolled in the course, and only seven opted for the hybrid virtual lab format. However we have controlled for factors such as the instructor and TA. All 23 students in the analysis had the same instructor and TA. The lab quiz average analysis suggest that students opting for the hybrid lab option are not at a disadvantage in reinforcing knowledge and understanding of lab content. The analysis of overall lab scores suggest that students in the hybrid virtual lab option are not disadvantaged in developing practical research skills. However, it would be premature to suggest that the hybrid virtual lab format is more effective than the conventional laboratory. It would be ideal to have better matched cohorts (equal numbers as opposed to $7: 16$ ) completing the hybrid virtual lab and conventional lab students in order to better control for factors that might bear on student performance, following a similar approach to a study that also investigated virtual labs [9]. It should be noted that this offering of the hybrid virtual lab was not designed as a test for the format, but rather the offering provided an opportunity to assess its effectiveness, and this is reflected in such study design deficiencies as small sample size and imbalanced cohorts.

An interesting observation to be made is that fewer students in BLG 143 exhibited interest in the virtual lab option than the conventional format. This was a surprise when the offering was made, as we expected many students would be interested in the out-of-lab format and the flexibility it offered. The students that did opt for the virtual lab format may have done so for a variety of reasons, but had confidence in their own abilities to independently learn concepts presented in the lab. 


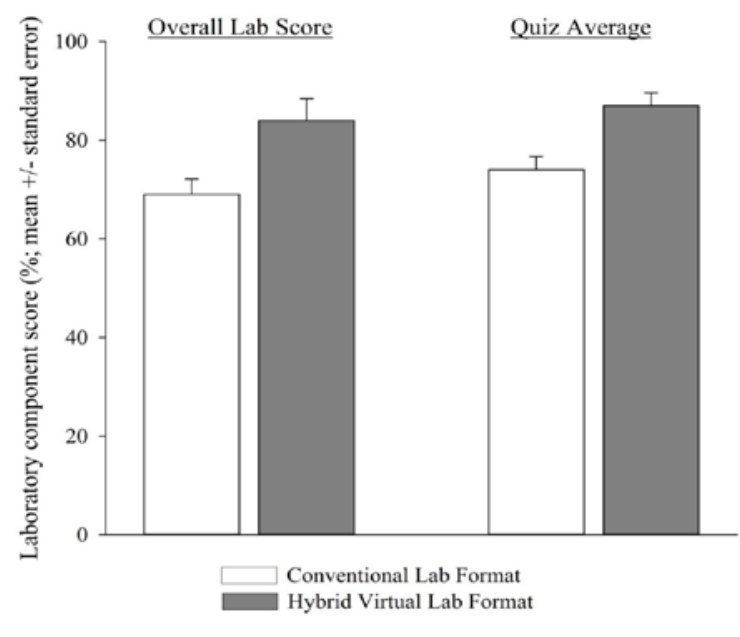

Figure 1. Overall lab performance (composite score) and laboratory quiz average for BLG 143 during the Winter, 2015 transition offering

Under the ideal circumstances, studies have shown that some virtual labs have been found to save students' time [17].

At first glance, the virtual labs appear to be a net loss in funds with $\$ 0$ cost to the student and a $\$ 40$ cost to assemble each kit. Multiple studies suggest that virtual labs can combat budget cuts due to the reduced spending on staff and equipment [6], [7], [10]. This is evident for the Ryerson University virtual labs since the gain in funding is the net amount after accounting for the reduced money being spent on laboratory space and personnel. There were 33 lab sections in fall 2014 for BLG 143, one TA and one lab space accommodated two-three sections. The conventional labs during the fall semester had to run throughout the entire week at multiple times during the day. A higher amount of students who opt-into the virtual lab may allow materials to be requested at bulk, slightly lowering the cost of kits. In addition to this, the take-home nature of the lab would reduce the number of students in the lab space, to a point where entire bookings may no longer be required. In addition to this, the virtual labs generally require less teaching support where the conventional lab needed the TA to conduct a pre-lab talk and supervise students for three hours. The virtual lab depends on video demonstrations and can have one to two hours of virtual office hours. Given these points, implementing virtual labs at a larger scale may provide financial relief from paying for TAs and lab space.

The virtual lab addresses different learning outcomes and styles as described by the Bloom's Taxonomy of Cognitive Learning Outcomes and Gardner's Multiple Intelligences. Bloom's Taxonomy is a model used to identify common educational learning outcomes [18]. It was also used to determine how well the Ryerson University laboratory exercises incorporated these outcomes into the learning material. Lab quiz questions focused on knowledge and comprehension of material, supplemented by the lab report sheets for each module which aided student comprehension of concepts learned throughout the experiment. Having students prepare and write a formal lab report provided a chance for them to apply the knowledge they gained while conducting the experiment while also analyzing their results to form conclusions. The lab modules included a few questions within, written to develop curiosity in the student such as creating null and alternate hypotheses for the effect of $\mathrm{pH}$ on amylase. In this assessment, students used their acquired knowledge to develop a plan and test their hypotheses either in reality or mentally in order to draw conclusions on the collected data. The entire laboratory module focused on protein structure and factors which affect structure, where the student can synthesize the information in earlier sections and extrapolate that into answering why anthocyanin pigments change colour as a function of $\mathrm{pH}$.

In addition, the different learning styles of students as described by Gardener's theory of multiple intelligences are also targeted with each lab.

A linguistic mind was developed through communication skills in the form of lab reports, critical thinking questions. On the other hand, a student with a numerical mindset was stimulated through various questions requiring a logical line of reasoning or mathematical calculations, such as solving genetic problems with Punnett squares (Lab 5). A visual-spatial learner benefitted from the virtual lab because video demonstrations and observable changes were commonplace for most experiments. Also, a bodily-kinesthetic learner had a complete lab kit (see Table 2) to manipulate and the opportunity to use a compound microscope when learning new concepts (Lab 3). A student whose style most resembles a naturalist had the freedom to learn the lab material outside the confines of a physical laboratory. They may have enjoyed the ability to choose a surrounding with natural elements as opposed to one with lab benches and equipment. Finally, musically minded students often study or learn better while listening to music. The take-home nature of the alternative lab allowed them to do so, which would be unlikely to occur in the conventional lab.

A common question hybrid virtual lab students raised was whether they were allowed to work with their fellow peers. Isolation of students was not prescribed for either format, where students had the freedom to work as a group or independently, corresponding to interpersonal and intrapersonal styles. While a student may have worked completely independently through the laboratory exercises, for Lab 3 (see Table 1) students were required to attend an in-lab session to handle a compound microscope 
in addition to using Smart Microscope app (www.smartmicrophone.net). This provided them with the opportunity to work with their peers and to discuss material with each other. Students are key drivers in directing their learning approach.

While students enrolled in both versions of the lab had access to the instructor through both email and weekly three hour sections separate from the laboratory. It is noted that future iterations of the virtual lab would have virtual office hours setup for students. To address this, virtual lab students would have dedicated time to ask questions and receive answers to their instructor (via chat room, forum, or discussion board) providing them with an additional resource outside of the videos and manual.

\section{Conclusions}

Future studies of the Ryerson University approach to virtual labs should seek to obtain more quantitative data in order to make more meaningful connections between student performance and the effects of laboratory delivery format. This alternative version of the lab, lead to the enhancement of both curricula by following benefits and addressing problems faced by the other party offering two modes of the lab is that students are trained to acquire research skills rather than laboratory skills. Hybrid virtual labs give students the opportunity to learn content and complete tasks at their own pace, which follow the logic of the virtual lab providing convenience to students as opposed to the schedule of conventional labs.

\section{References}

[1] de Jong, T., Linn, M. C., \& Zacharia, Z. C. (2008). Physical and Virtual laboratories in science and engineering education. Science, 340(6130), 305-308.

[2] Huang, C. (2004). Virtual Labs: E-Learning for Tomorrow. PLoS Biology, 2(6), 734-735.

[3] Waldrop, M. M. (2013). Education online: the virtual lab. Nature, 499(7458), 268-270.

[4] Sauter, M., Uttal, D. H., Rapp, D. N., Downing, M., \& Jona, K. (2013). Getting real: the authenticity of remote labs and simulations for science learning. Distance Education, 34(1), 37-47.

[5] Tatli, Z., \& Ayas, A. (2010). Virtual laboratory applications in chemistry education. Procedia - Social and Behavioral Sciences, 9, 938-942.

[6] Dobson, J. L. (2009). Evaluation of the Virtual Physiology of Exercise Laboratory program. Advances in Physiology Education, 33(4), 335-342.

[7] Darrah, M., Humbert, R., Finstein, J., Simon, M., \& Hopkins, J. (2014). Are Virtual Labs as Effective as
Hands-on Labs for Undergraduate Physics? A Comparative Study at Two Major Universities. Journal of Science Education and Technology, 23(6), 803-814.

[8] Flowers, L. O. (2011). Investigating the Effectiveness of Virtual Laboratories in an Undergraduate Biology Course. Journal of Human Resources \{ \&\} Adult Learning, 110-116.

[9] Swan, A. E., \& O’Donnell, A. M. (2009). The contribution of a virtual biology laboratory to college students' learning. Innovations in Education and Teaching International, 46(4), 405-419.

[10] Scheckler, R. K. (2003). Virtual labs: A substitute for traditional labs? International Journal of Developmental Biology, 47(2-3), 231-236.

[11] Meir, E., Perry, J., Stal, D., Maruca, S., \& Klopfer, E. (2005). How effective are simulated molecular-level experiments for teaching diffusion and osmosis? Cell Biology Education, 4(3), 235-248.

[12] Weisman, D. (2010). Incorporating a collaborative web-based virtual laboratory in an undergraduate bioinformatics course. Biochemistry and Molecular Biology Education, 38(1), 4-9.

[13] Tatli, Z., \& Ayas, A. (2013). Effect of a Virtual Chemistry Laboratory on Students 'Achievement. Educational Technology \{\&\} Society, 16(1), 159-170.

[14] Stuckey-Mickell, T. a., \& Stuckey-Danner, B. D. (2007). Virtual labs in the online biology course: student perceptions of effectiveness and usability. MERLOT Journal of Online Learning and Teaching., 3(2), 105-111.

[15] Limniou, M., Papadopoulos, N., Giannakoudakis, A., Roberts, D., \& Otto, O. (2007). The integration of a viscosity simulator in a chemistry laboratory. Chemistry Education Research and Practice, 8(2), 220.

[16] Tulbure, C. (2011). Do different learning styles require differentiated teaching strategies? Procedia Social and Behavioral Sciences, 11, 155-159.

[17] Pyatt, K., \& Sims, R. (2011). Virtual and Physical Experimentation in Inquiry-Based Science Labs: Attitudes, Performance and Access. Journal of Science Education and Technology, 21(1), 133-147.

[18] Adams, N. E. (2015, July). Bloom's taxonomy of cognitive learning objectives. Journal of the Medical Library Association: JMLA. 65 East Wacker Place, Suite 1900, Chicago, IL 60601-7246.

\section{Acknowledgements}

We are grateful to the undergraduate class of BLG 143 2014-2015 for their participation. We are thankful to Karen J. Puddephatt for her technical support. 